Research Article

\title{
Cascade Failure Model in Multimodal Transport Network Risk Propagation
}

\author{
Zhenggang He, Jing-Ni Guo $(\mathbb{D}$, and Jun-Xiang Xu $(\mathbb{D}$ \\ Southwest Jiaotong University, Chengdu, China \\ Correspondence should be addressed to Jing-Ni Guo; 947082371@qq.com
}

Received 22 May 2019; Revised 27 July 2019; Accepted 29 July 2019; Published 6 December 2019

Academic Editor: Alessio Ishizaka

Copyright ( 92019 Zhenggang He et al. This is an open access article distributed under the Creative Commons Attribution License, which permits unrestricted use, distribution, and reproduction in any medium, provided the original work is properly cited.

\begin{abstract}
The cascade failure theory is introduced into the risk propagation problem of the multimodal transport network in order to study the inherent law of risk propagation and provide theoretical support for the safety management of multimodal transport networks. Firstly, this paper analyses the characteristics of the multimodal transport network and concludes that the risk of the multimodal transport network belongs to failure risk. Secondly, the applicability of cascade failure theory is expounded. Based on cascade failure theory, a risk propagation model of the multimodal transport network is established. Through simulation experiments, the risk propagation of the multimodal transport network is analyzed from the differences of node distribution and node type. The process is analyzed, and the results show that different node distributions and different types of risk source nodes will have an impact on the risk propagation process. The influence of four types of node distributions on the risk propagation effect is in the following order: increasing type $>$ concave-convex type $\approx$ balanced type $>$ decreasing type. The influence of four types of source nodes on the risk propagation effect is in the following order: transportation type $>$ transporting type $>$ storage type $>$ assistant type.
\end{abstract}

\section{Introduction}

Multimodal transport is a mode of transport that China is focusing on. It plays an important role in the development of the country. Multimodal transport refers to the effective connection of two or more modes of transport to provide the whole process of integrated organization of cargo transport services. Multimodal transport network is a complex network involving transport nodes, transport connecting edges, transshipment nodes, and transshipment connecting edges. Each node contains different safety risks, which together constitute the safety risk system of the multimodal transport network. In the safety risk system, the phenomenon of risk propagation is widespread. With the increase of network complexity, the degree of association between nodes becomes stronger. Once a node fails, the risk will spread rapidly to the associated nodes, which will cause multiple node failures in the multimodal transport network, causing network paralysis, affecting the speed and quality of cargo transport, and affecting the normal operation of social economy. Therefore, the study of the risk propagation law of the multimodal transport network can understand the speed and direction of risk propagation in the multimodal transport network, which is conducive to the control of security risk of multimodal transport, the reduction of network node inefficiency after failure, and the effective management of multimodal transport networks.

For the study of multimodal transport, scholars mainly focus on the optimization of the transport organization mode of multimodal transport [1-6], the layout of facilities and stations $[7,8]$, policies, laws, and regulations [9-12], and related technical applications [13-16]. At present, the cascade failure problem of the multimodal transport network is still blank, but there are plenty of studies on urban transport networks. Zhong and Shuai [17] established a cascade failure model of the dangerous goods transportation network under serial attack and took the node survival rate and relative secondary cascade failure as the evaluation factors. Liu et al. [18] proposed a cascade failure model of the urban traffic network under emergencies, and the initial load was determined by the incremental loading method under multipath probability. Peng [19] constructed a cascade model of 
the urban road traffic network based on the disaster propagation dynamics, with the number of collapsed nodes and the node repair rate as the evaluation factors. Zhang et al. [20] analyzed the influence of different factors on the network and obtained the importance of different factors affecting the cascade failure process in the traffic network. Qian et al. [21] studied the effects of time delay and selfhealing on cascade failure in traffic congestion. In a multimodal transport network, nodes no longer refer to the intersection of roads but the intersection of different modes of transport. Compared with urban transport networks, multimodal transport networks have poorer connectivity and stronger correlation between nodes. After the node fails, the effect of the failure will be more intense. Therefore, it is of great practical significance to study the cascade failure of the multimodal transport network.

In terms of methods, for the study of cascade failure theory, Wang and Rong [22] studied the impact of the initial load strength of different nodes on the network under the two attack modes of the US grid. Cao et al. [23] compared the effects of three link-adding strategies to improve network robustness in the network structure, and the results show that the low-polarization linking strategy is most effective. Jiang and $\mathrm{Ma}$ [24] believed that the key for improving network robustness against cascading failures is to maximally defend cascade propagation with minimum total extracapacity of all nodes and discussed three general extracapacity distributions including degree-based distribution (DD), average distribution (AD), and random distribution (RD). They proposed a novel SC-based local load redistribution mechanism to improve the cascade defense ability of the network and confirmed the effectiveness of the SC local load redistribution method. Jiang et al. [25] proposed two extracapacity distributions including degreebased distribution and average distribution and compared the effects of four heuristic algorithms to discover key sets of nodes in the network, including the high-degree-first (HDF) algorithm, low-degree-first (LDF) algorithm, random algorithm (RA), and greedy algorithm (GA). The results show that the greedy algorithm can efficiently find the set of key nodes in both scale-free and random networks. The existing research results are based on the consistency of nodes in the network, and the initial load strength and node distribution rules of the network are studied. However, in the multimodal transport network, there are several different types of nodes corresponding to different attributes, which can cause different cascading failure effects when a node fails. The authors believe that the node attribute is similar to the initial load strength and node distribution rules, and it is the key factor affecting the cascading failure effect in the network. Therefore, it is a new research idea to study the impact of different attributes of nodes on the network.

\section{Risk Characteristic Analysis of Multimodal Transport Networks}

In the process of risk propagation in the network, different network topology structures will bring significant difference to the propagation process. Only when the power of risk propagation exceeds the threshold of the network, it can spread in the network [26]. According to the conclusion of Lei [27], the multimodal transport network can be approximated to the scale-free network. Because of the low propagation threshold in the scale-free network, network risk will be easier to spread.

According to a different nature of risk propagation, network risk can be divided into two types: infectious risk and failure risk [28]. Infectious risk refers to the risk that will spread between adjacent nodes with a certain probability and eventually lead to the infection of the whole network, such as food quality risk. Failure risk refers to that when the nodes in the network are affected by internal or external factors, the load on the failed nodes will transfer to the associated adjacent nodes, and the adjacent nodes may fail beyond their maximum load, which will lead to cascade failure so that the risk has an impact on the whole network, such as traffic congestion risk.

The multimodal transport network is a complex network composed of different transport agents, including multiple nodes, edges, and transshipment nodes. When the risk in the network affects the node, the node will absorb certain risk by its resistance. When it exceeds the safety threshold of the node, the node will fail. And the load on the failed node will transfer to the associated adjacent node. The adjacent node may fail because of the increase of the load and exceed the maximum load, and then the risk will be propagated downward, which makes the risk affect the whole network. Therefore, it can be considered that the risk characteristics of the multimodal transport network are similar to those of failure type and belong to failure risk.

\section{Applicability Analysis of Cascade Failure Theory}

In this paper, the load-capacity model in cascade failure theory is adopted, which includes the node failure model [29], edge failure model, and node-edge hybrid failure model. It represents cascade failure phenomena caused by different failure sources and can quantify failure risk propagation phenomena in multimodal transport networks comprehensively. This paper uses the node failure model to study.

The characteristics of the multimodal transport network are compared with those of cascade failure theory, as shown in Table 1.

From this table, we can see that the characteristics of the multimodal transport network and cascade failure theory have obvious consistency. Therefore, cascade failure theory can be applied to the risk propagation of multimodal transport networks.

\section{Establishment of Risk Dissemination Model}

\subsection{Model Hypothesis}

(i) Each node in the multimodal transport network has a certain safety threshold, and the influence of internal and external environments within the safety threshold can be digested by the node itself. If the 
TABLE 1: Feature comparison.

\begin{tabular}{|c|c|c|c|c|c|}
\hline- & Composition & Risk types & Propagation process & $\begin{array}{c}\text { Propagation } \\
\text { subject }\end{array}$ & $\begin{array}{l}\text { Risk } \\
\text { resistance }\end{array}$ \\
\hline $\begin{array}{l}\text { Multimodal transport } \\
\text { network }\end{array}$ & $\begin{array}{c}\text { Nodes, edges, and transshipment } \\
\text { nodes }\end{array}$ & $\begin{array}{l}\text { Failure } \\
\text { risk }\end{array}$ & $\begin{array}{l}\text { Adjacent nodes/ } \\
\text { edges }\end{array}$ & Cargo & Yes \\
\hline Cascade failure theory & Nodes and edges & $\begin{array}{l}\text { Failure } \\
\text { risk }\end{array}$ & $\begin{array}{c}\text { Adjacent nodes/ } \\
\text { edges }\end{array}$ & Load & Yes \\
\hline
\end{tabular}

safety threshold is exceeded, the failure risk will be brought.

(ii) Each node has a certain self-repairing ability. When affected by the risk, the node can resolve the risk through self-repairing.

(iii) According to a different nature of node operation, it can be divided into the transport node, storage node, auxiliary node, and transshipment node.

(iv) In the process of risk propagation, the self-repairing function of nodes is not considered.

4.2. Model Establishment. In the multimodal transport network $G=(V, E)$, the set of nodes $V=\left\{v_{1}, v_{2}, \ldots, v_{n}\right\}$, the set of edges $E=\left\{e_{1}, e_{2}, \ldots, e_{m}\right\}$, the safety threshold of the node $i$ is $S_{i}$, and different types of nodes have different safety thresholds. When $t=0$, the initial load of the node $i$ is $L_{i}$ and the maximum load is $C_{i}$. The following formula can be obtained according to the ML model, in which $\beta$ is the variable parameter:

$$
C_{i}(0)=(1+\beta) L_{i}(0)
$$

Generally, the states of nodes can be divided into "normal," "overload," and "failure" [30]. When the node $v_{i}$ is affected by the internal and external factors, the difficulty of load passing through the node will change because of the change of the node state. In this paper, overload function $F_{i}(t)$ is used to express the difficulty of load passing through nodes:

$$
F_{i}(t)=\left\{\begin{array}{lc}
1, & L_{i}(t) \leq L_{i}(0), \\
1+\frac{L_{i}(t)-L_{i}(0)}{C_{i}-L_{i}(0)}(N-1), & L_{i}(0) \leq L_{i}(t) \leq C_{i}, \\
N, & L_{i}(t)>C_{i}, \\
N^{2}, & \text { failure. }
\end{array}\right.
$$

In multimodal transport networks, different states of nodes lead to different load allocation modes. When the node $v_{i}$ fails because of a fault, all loads on the node need to be redistributed and distributed to adjacent nodes according to the proportion of edge weights. Let $\omega_{i j}$ be the weight of the edge between $v_{i}$ and $v_{j}$ :

$$
L_{j}(t+1)=L_{j}(t)+\frac{\omega_{i j} L_{i}(t)}{\sum_{j=1}^{k_{i}} \omega_{i j}} .
$$

When the node $v_{i}$ is overloaded, the part of the node that exceeds the maximum load needs to be reallocated:

$$
L_{j}(t+1)=L_{j}(t)+\frac{\omega_{i j}\left(L_{i}(t)-C_{i}\right)}{\sum_{j=1}^{k_{i}} \omega_{i j}} .
$$

When the load exceeds the maximum load $C_{j}$ of the node $v_{j}$, the excess part will continue to propagate to the adjacent nodes until the load of all nodes in the network is within the maximum load, and the cascade failure ends.

In this paper, the proportion of abnormal nodes and average network efficiency are used to measure the effect of risk propagation in multimodal transport networks.

4.2.1. Proportion of Abnormal Nodes. Let the total number of nodes in the multimodal transport network be $N$, in which the total number of abnormal nodes is $N_{f}$ and the proportion of abnormal nodes is $R_{f}$. The higher the proportion, the stronger the risk propaganda in the multimodal transport network or otherwise the weaker the risk propaganda in the multimodal transport network:

$$
R_{f}=\frac{N_{f}}{N} .
$$

4.2.2. Average Network Efficiency. Average network efficiency $E$ is used to measure the connectivity of multimodal transport networks. When the nodes are in the abnormal state, the higher the average network efficiency is, the weaker the dissemination of risk in the network is or otherwise the stronger the risk propagation is. $d_{i j}$ represents the path with the highest load trafficability between node $v_{i}$ and node $v_{j}$, and $P(i, j)$ represents the set of all paths between node $v_{i}$ and node $v_{j}$.

$$
\begin{aligned}
E & =\frac{1}{N(N-1)} \sum_{i \neq j} \frac{1}{d_{i j}} \\
d_{i j} & =\min \left\{\sum_{p} F_{p}(t), \quad p \in P(i, j)\right\} .
\end{aligned}
$$

\section{Risk Propagation Simulation}

5.1. Simulation Process. In this paper, the effect of risk propagation in multimodal transport networks is analyzed from the difference of node distribution and node type, and the node types include transshipment, storage, auxiliary, and transportation. Different types of nodes have different 


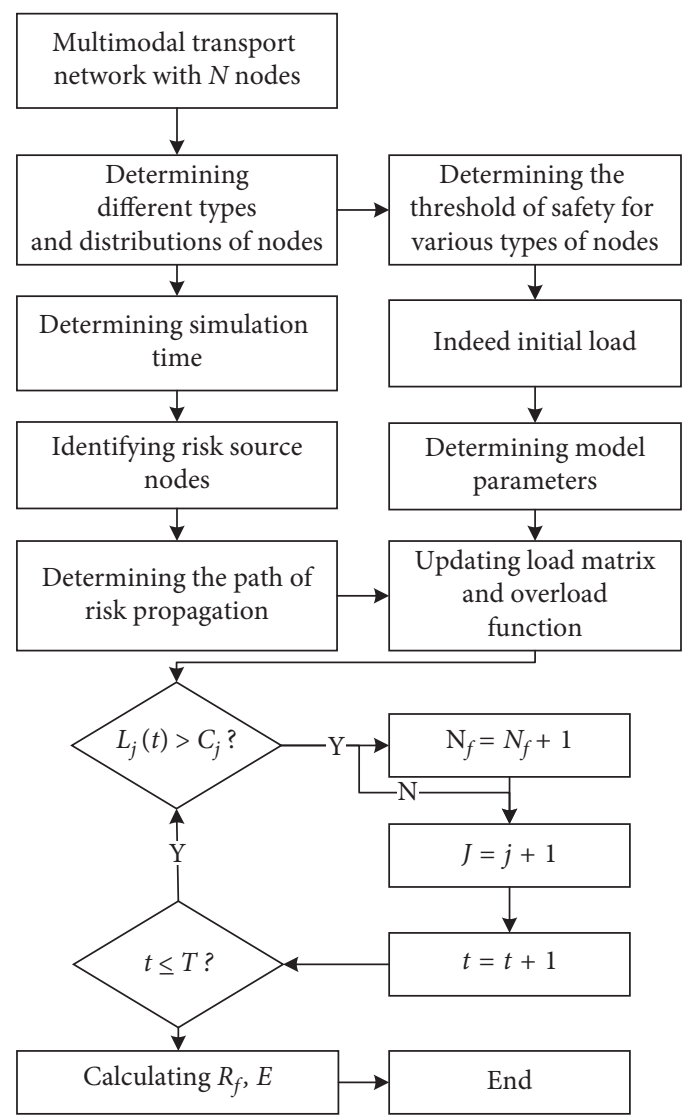

FIgURE 1: Simulation process.

TABle 2: Node parameter considerations.

\begin{tabular}{|c|c|c|c|c|}
\hline Node type & Effects & Facilities and equipment & Redundancy & Expansion measures \\
\hline Auxiliary node & $\begin{array}{l}\text { Used for loading, unloading, } \\
\text { handling, distribution processing, } \\
\text { and information processing of } \\
\text { goods in multimodal transport } \\
\text { networks }\end{array}$ & $\begin{array}{l}\text { Software and hardware facilities } \\
\text { such as forklifts, work vehicles, } \\
\text { conveyors, and information } \\
\text { admission systems }\end{array}$ & Little & $\begin{array}{l}\text { Accelerating operational } \\
\text { efficiency }\end{array}$ \\
\hline Storage node & Used for the storage of goods & $\begin{array}{c}\text { Hardware facilities for pallets and } \\
\text { shelves }\end{array}$ & Normal & $\begin{array}{c}\text { Utilization of temporary storage } \\
\text { areas }\end{array}$ \\
\hline $\begin{array}{l}\text { Transshipment } \\
\text { node }\end{array}$ & $\begin{array}{l}\text { Used for loading, unloading, } \\
\text { handling, etc. of goods at the } \\
\text { intersection of two modes of } \\
\text { transportation }\end{array}$ & $\begin{array}{l}\text { Hardware facilities for cranes and } \\
\text { conveyors }\end{array}$ & Much & $\begin{array}{l}\text { Infrastructure equipment between } \\
\text { different modes of transport can } \\
\text { be used with each other }\end{array}$ \\
\hline Transport node & Transport operation for goods & Transport vehicles & More & $\begin{array}{l}\text { Meeting the transportation } \\
\text { demand by adjusting the number } \\
\text { of transport vehicles }\end{array}$ \\
\hline
\end{tabular}

maximum load values. The node states include normal, overload, and failure. The simulation process of risk propagation in multimodal transport networks is shown in Figure 1.

5.2. Analysis of Simulation Results. In this paper, the total number $N=500$ of multimodal transport network nodes is set. Based on the field investigation and questionnaire survey of multimodal transport stations, the parameters of different types of nodes can be set according to the factors shown in Table 2 .

Considering the factors in Table 2, we can get that the parameters $\beta$ of auxiliary, storage, transshipment, and transport nodes are $0.1,0.3,0.5$, and 0.7 , respectively. Different proportions of failure nodes are set up to simulate the multimodal transport network under different conditions, and the risk propagation law in the multimodal transport network can be studied from many aspects and perspectives.

5.2.1. Analysis of Risk Dissemination Effects under Different Node Distributions. In multimodal transport networks, different functional preferences can lead to a diverse distribution of node types. The initial load values of auxiliary, storage, transshipment, and transport nodes are the same 


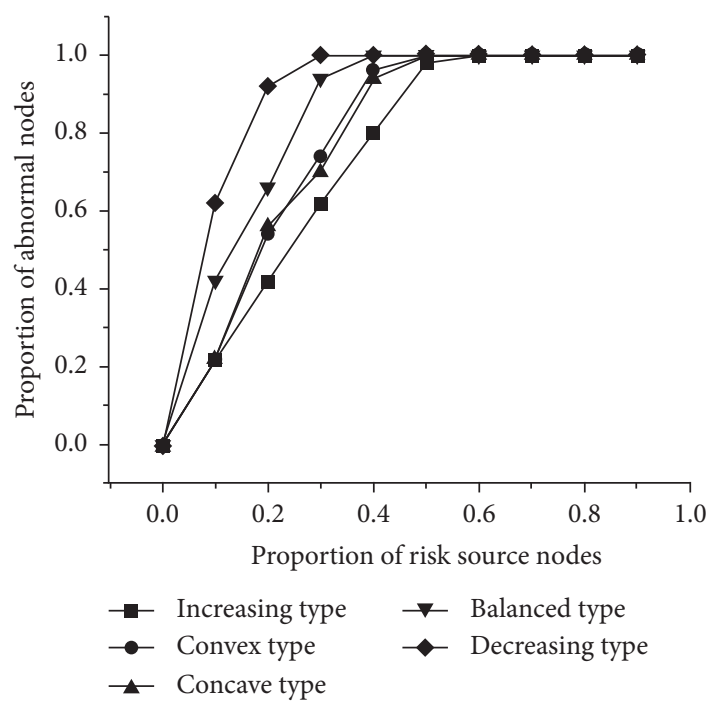

Figure 2: Proportion of abnormal node states with different node distributions.

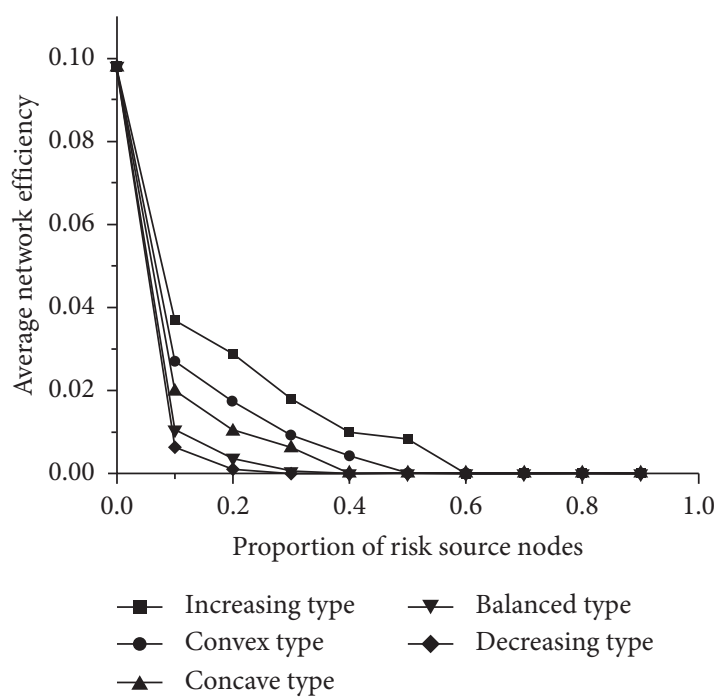

Figure 3: Average network efficiency with different node distributions.

under the stable state of the network. According to different proportions of nodes in the multimodal transport network, the distribution of nodes can be expressed as the balanced type (auxiliary node:storage node:transshipment node: transport node $=1: 1: 1: 1)$, increasing type $(1: 2: 3: 4)$, decreasing type $(4: 3: 2: 1)$, convex type $(1: 2: 2: 1)$, and concave type $(2: 1: 1: 2)$.

From Figures 2 and 3, it can be seen that the multimodal transport network with different node distributions presents different proportions of abnormal nodes and average network efficiency. When the proportion of risk source nodes is more than $60 \%$, the multimodal transport network presents a complete failure state. At this time, the nodes of the whole network fail or are overloaded, and the average network efficiency is almost 0 . When the proportion of risk source nodes is less than $60 \%$, the order of the proportion of abnormal nodes from large to small is increasing

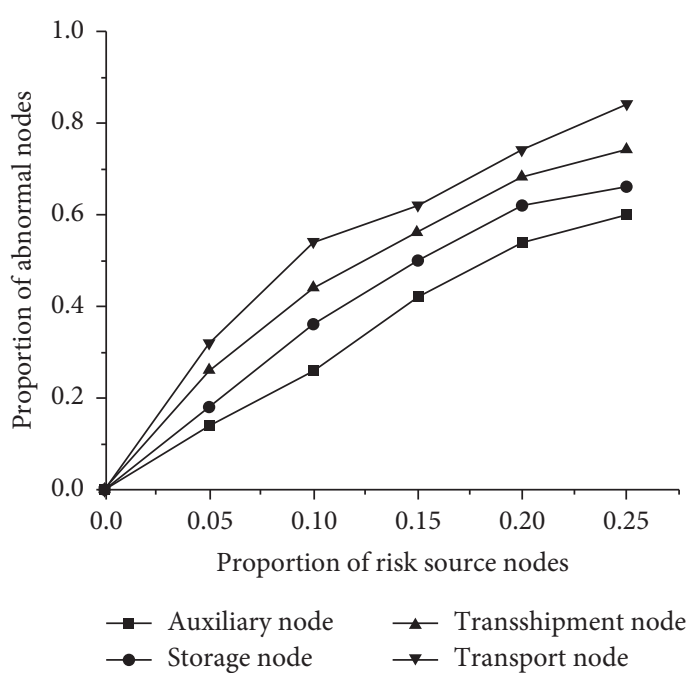

FIGURE 4: Proportion of abnormal node states with different node types.

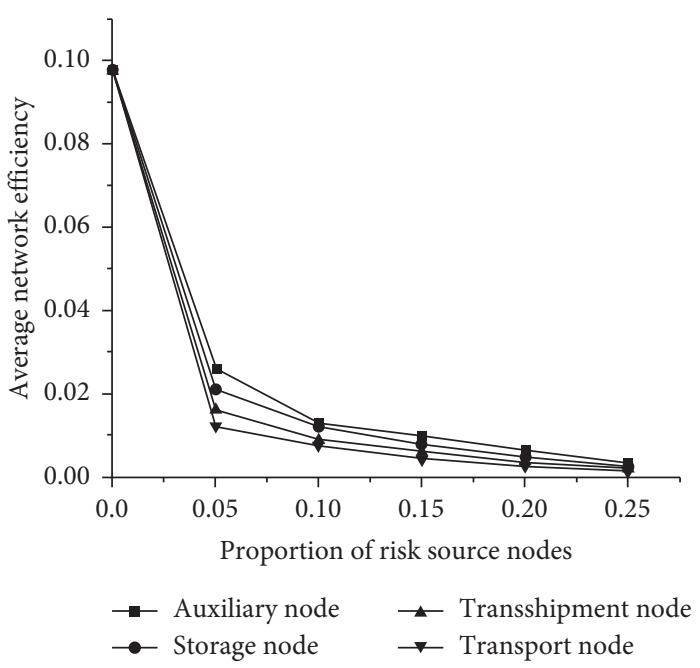

Figure 5: Average network efficiency with different node types.

type $>$ concave type $>$ convex type $>$ balanced type $>$ decreasing type. Meanwhile, the order of average network efficiency is the same as that of abnormal nodes. Therefore, in the multimodal transport network, the risk spreading range of the increasing network node distribution is smaller, and the risk spreading range of the diminishing network node distribution is larger. It can be considered that the increasing network node distribution has higher stability, while the diminishing network node distribution has the opposite.

5.2.2. Effect Analysis of Risk Propagation under Different Types of Risk Source Nodes. Different types of risk source nodes will also affect the risk propagation effect in multimodal transport networks. Under the condition of balanced network node distribution, if the risk source is set as a single similar node, the proportion of abnormal nodes and the average network efficiency can be obtained, as shown in Figures 4 and 5 . 
Under the distribution of balanced network nodes, the proportion of each type of the risk source node varies from 0 to 0.25 . From Figures 4 and 5, when other conditions are the same, different types of risk source nodes will bring different risk propagation effects. It can be seen that the order of the proportion of abnormal state nodes is transport type$>$ transshipment type $>$ storage type $>$ auxiliary type, and the order of average network efficiency is the same. It shows that different types of risk source nodes will affect the risk transmission effect of the multimodal transport network and only its change range, but will not change its trend. In the multimodal transport network, the state change of the transport node has the greatest impact on the risk propagation in the whole network, and the auxiliary node has the least impact.

\section{Conclusion}

In this paper, the cascade failure model is applied to the multimodal transport network, and risk propagation in the multimodal transport network is studied. By establishing a mathematical model, the effect of risk propagation is evaluated by the proportion of abnormal nodes and the average network efficiency. The simulation results show the following:

(1) Different node distributions will affect the risk propagation effect of multimodal transport networks. When the proportion of risk source nodes is more than $60 \%$, all the nodes in the multimodal transport network are in a state of failure or overload. The average network efficiency is almost 0 , and the network completely fails. When the proportion of risk source nodes is less than $60 \%$, the stability of the increasing network is the highest, and the diminishing network is the most unstable. The impact on the effect of risk propagation from large to small is increasing $>$ concave $>$ convex $>$ balanced $>$ diminishing.

Different types of risk source nodes will also affect the risk propagation effect of multimodal transport networks. When the network fails completely, the failure of the transport node will cause a wider risk propagation in the same proportion of risk source nodes, while the failure of the auxiliary node will have the smallest impact, and the impact on the effect of risk propagation from large to small is transport $>$ transshipment $>$ storage $>$ auxiliary.

\section{Data Availability}

All data are available from the corresponding author upon request.

\section{Conflicts of Interest}

The authors declare that they have no conflicts of interest.

\section{References}

[1] A. Lozano and G. Storchi, "Shortest viable path algorithm in multimodal networks," Transportation Research Part A: Policy and Practice, vol. 35, no. 3, pp. 225-241, 2001.
[2] X.-F. Wang, L. I. Xiang, and C. H. E. N. Guan-Rong, Complex Network Theory and Its Application, Tsinghua University Press, Beijing, China, 2006.

[3] H. I. Calvete, C. Galé, M.-J. Oliveros, and B. SánchezValverde, "A goal programming approach to vehicle routing problems with soft time windows," European Journal of Operational Research, vol. 177, no. 3, pp. 1720-1733, 2007.

[4] X. Wang, Z.-B. Chi, and G. E. Xian-Long, "Research and analysis of vehicle multimodal transport model with time window," Computer Applied Research, vol. 28, no. 2, pp. 563-565, 2011.

[5] Y. Jiang, X.-C. Zhang, and Y.-L. Wang, "Cross-entropy method for choosing multimodal transport schemes," Transportation System Engineering and Information, vol. 12, no. 5, pp. 20-25, 2012.

[6] F.-L. Feng and Q.-Y. Zhang, "Environment-based optimization model for collaborative organization of multimodal transport modes," Journal of Railway Science and Engineering, vol. 9, no. 6, pp. 95-100, 2012.

[7] H. Qi, "Planning of container multimodal transport freight station based on fuzzy cluster Analysis," Journal of Fuqing Branch of Fujian Normal University, no. 5, pp. 13-16, 2010.

[8] L. I. A. O. Li-Ping, Research on Coordination of Layout and Capacity of Railway Container Terminal, Southwest Jiaotong University, Chengdu, China, 2012.

[9] C. Wang, "A study on the localization of damage to goods in multimodal transport," Legal System and Society, no. 33, p. 118, 2010.

[10] Q.-xing Xu, Study on the Legal Liability of Carriers in International Multimodal Carriage of Goods, Tianjin University of Finance and Economics, Tianjin, China, 2009.

[11] X. U. Zhan-Ping, A Comparative Study on the Liability System of International Multimodal Transport Operators, East China University of Politics and Law, Shanghai, China, 2011.

[12] B. Yang, Research on Civil Liability of Multimodal Transport Contract in China, Southwest University of Finance and Economics, Chengdu, China, 2010.

[13] S.-L. Huang and S.-Y. Liu, "Multimodal transport logistics decision SupportSystem based on distributed caching mechanism and data warehouse," Storage, Transportation and Maintenance of Commodities, vol. 30, no. 4, pp. 17-19, 2008.

[14] X. Sun and M. Meng, "Modern management technology in container transport and multimodal transport," Containerization, vol. 20, no. 8, pp. 10-12, 2009.

[15] P.-H. Sun, Research on Logistics Information Collection and Early Warning System of Container Multimodal Transport, Xi'an University of Architectural Science and Technology, Xi'an, China, 2011.

[16] X.-Y. Zhang and H.-D. Zhou, "Design of multimodal transport information system based on sensor network," Computer Technology and Development.vol. 22, no. 3, pp. 199-202, 2012.

[17] P.-Y. Zhong and B. Shuai, "Model of cascading failure in hazardous materials transportation network under series of terrorist attacks," Systems Engineering-Theory \& Practice, vol. 34, no. 4, pp. 1059-1065, 2014.

[18] W. Liu, K.-Q. Chen, Y.-Y. LIU et al., "Cascading failure simulation of road network based on prospect theory," Journal of Transportation Systems Engineering and Information Technology, vol. 18, no. 01, pp. 145-151, 2018.

[19] S. Peng, The Research on Disaster Spreading Dynamics Model for Cascading Failure for Urban Road Traffic Network, Changsha University of Science \& technology, Changsha, China, 2014. 
[20] Y. Zhang, Y. Lu, G. Lu, P. Chen, and C. Ding, "Analysis of road traffic network cascade failures with coupled map lattice method," Mathematical Problems in Engineering, vol. 2015, no. 4, Article ID 101059, 8 pages, 2015.

[21] Y. Qian, B. Wang, Y. Xue, J. Zeng, and N. Wang, "A simulation of the cascading failure of a complex network model by considering the characteristics of road traffic conditions," Nonlinear Dynamics, vol. 80, no. 1-2, pp. 413-420, 2015.

[22] J.-W. Wang and L.-L. Rong, "Cascade-based attack vulnerability on the US power grid," Safety Science, vol. 47, no. 10, pp. 1332-1336, 2009.

[23] X.-B. Cao, C. Hong, W.-B. Du, and J. Zhang, "Improving the network robustness against cascading failures by adding links," Chaos, Solitons \& Fractals, vol. 57, pp. 35-40, 2013.

[24] Z. Jiang and J. Ma, "An efficient local cascade defense method in complex networks," International Journal of Modern Physics C, vol. 28, no. 3, article 1750031, 2016.

[25] Z.-Y. Jiang, Z.-Q. Liu, X. He, and J.-F. Ma, "Cascade phenomenon against subsequent failures in complex networks," Physica A: Statistical Mechanics and Its Applications, vol. 499, pp. 472-480, 2018.

[26] B. Á Marián and P. S. Romualdo, "Epidemic spreading in correlated complex networks," Physical Review E Statistical Nonlinear \& Soft Matter Physics, vol. 66, no. 4, p. 47104, 2002.

[27] K. Lei, Research on Risk Spread and Control Strategy of Multimodal Transportation network, Beijing Jiaotong University, Beijing, China, 2016.

[28] X. Wang, Agri-food Supply Chain Modeling and Risk Propagation Based on Complex Network theory, Jilin University, Changchun, China, 2017.

[29] Y. Moreno, J. B. Gómez, and A. F. Pacheco, "Instability of scale-free networks under node-breaking avalanches," Europhysics Letters (EPL), vol. 58, no. 4, pp. 630-636, 2002.

[30] S. Gao, Research on Invulnerability of Wuhan Rail Transshipment Network under Cascading failure, Wuhan University of Technology, Wuhan, China, 2017. 


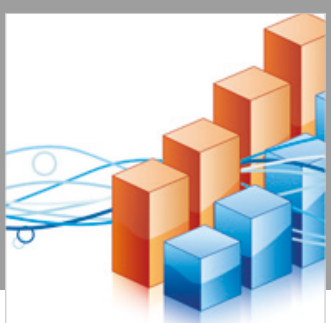

Advances in

Operations Research

\section{-n-m}
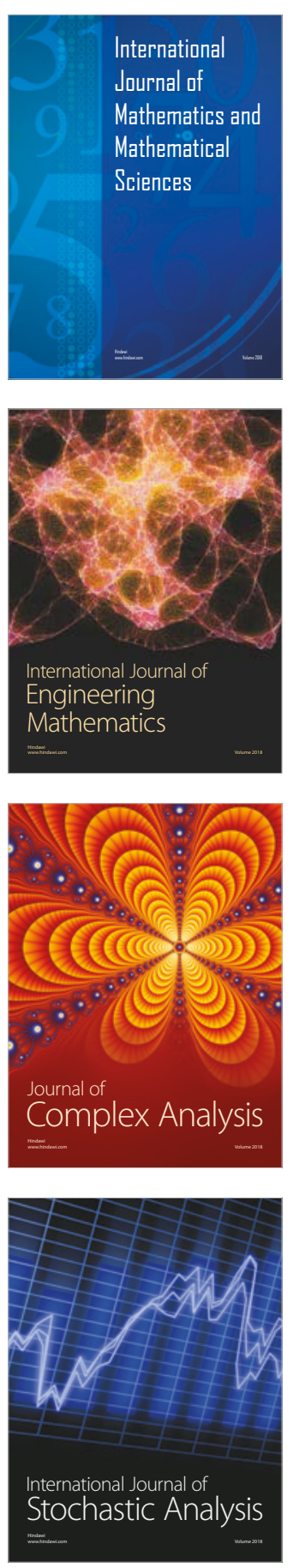
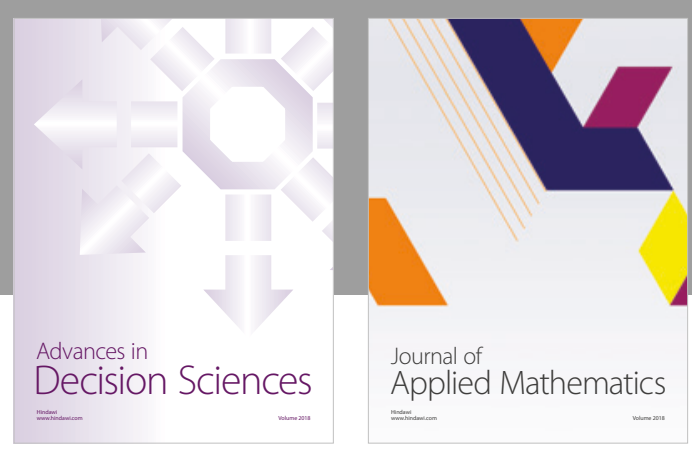

Journal of

Applied Mathematics
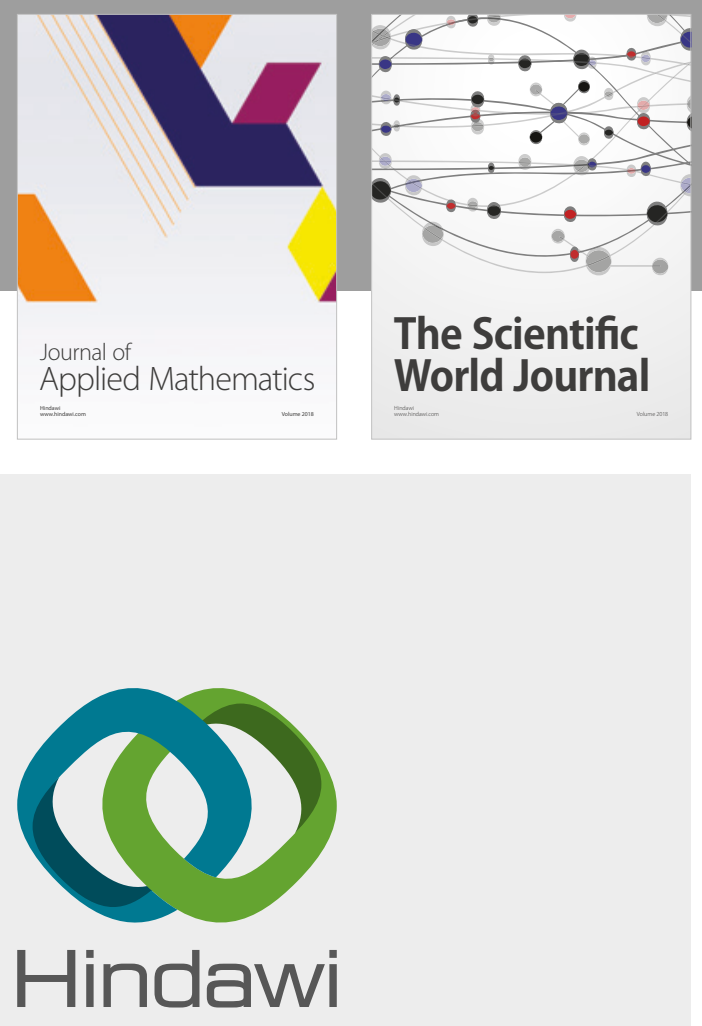

Submit your manuscripts at

www.hindawi.com

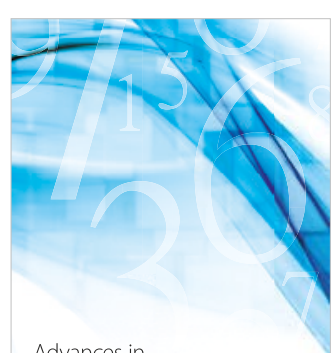

Advances in
Numerical Analysis
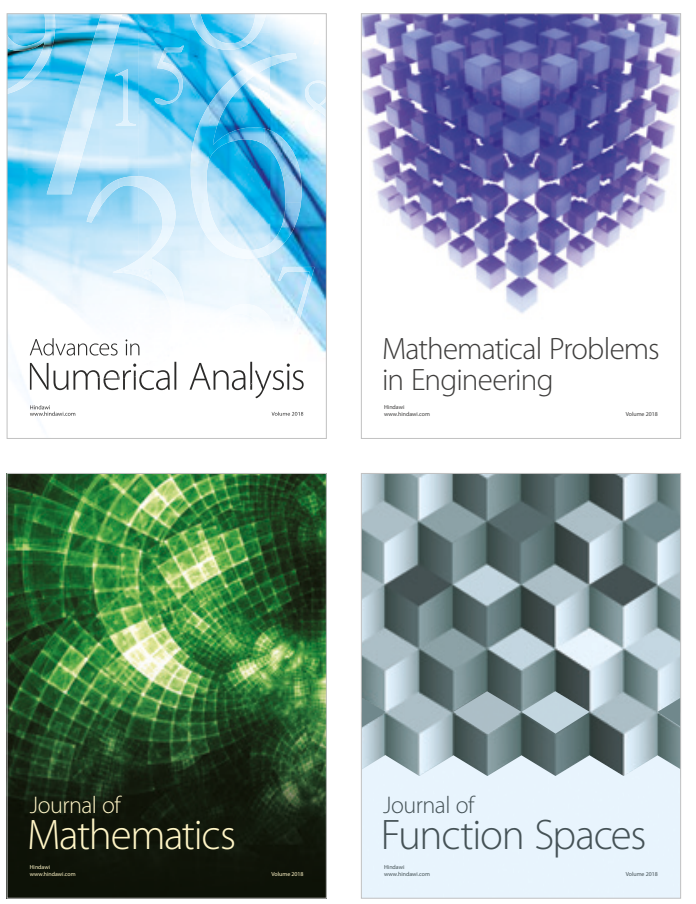

Mathematical Problems in Engineering

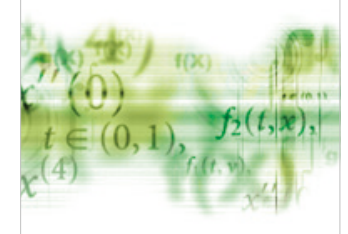

International Journal of

Differential Equations

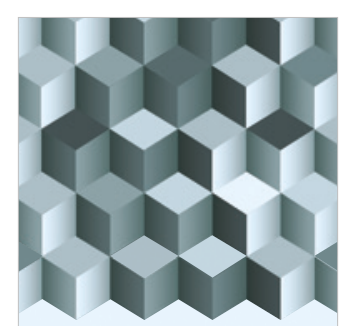

Journal of

Function Spaces

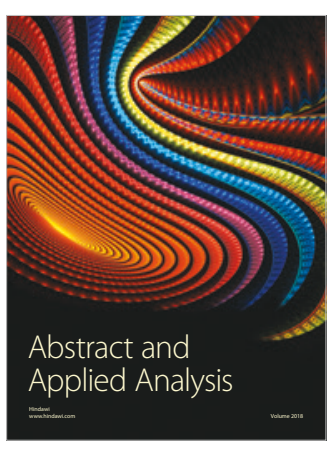

The Scientific

World Journal

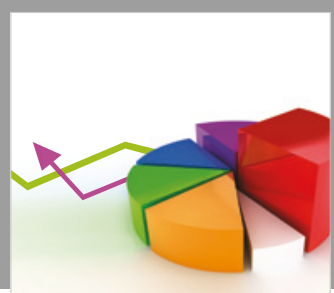

Journal of

Probability and Statistics
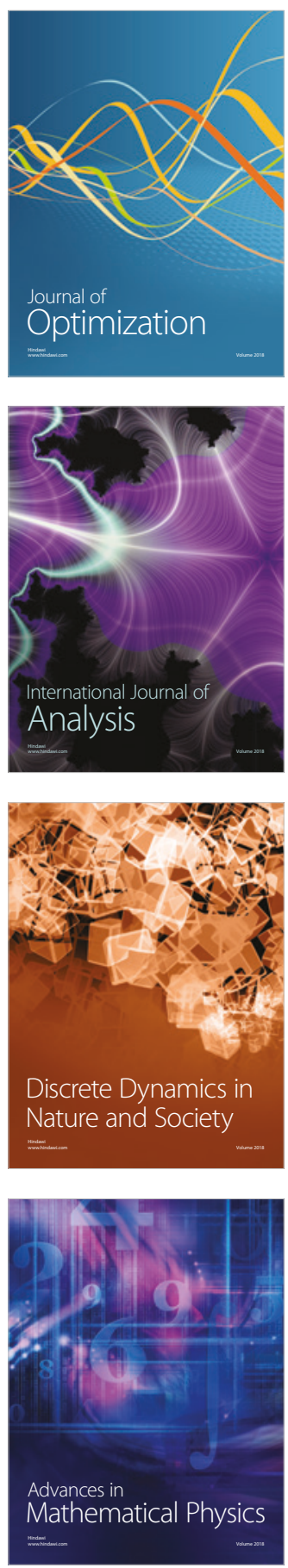